\title{
The Parameterisation of Scalar Transfer over Rough Ice
}

\author{
C. J. P. P. Smeets • M. R. van den Broeke
}

Received: 24 May 2007 / Accepted: 3 July 2008 / Published online: 29 July 2008

(C) The Author(s) 2008

\begin{abstract}
We test a surface renewal model that is widely used over snow and ice surfaces to calculate the scalar roughness length $\left(z_{s}\right)$, one of the key parameters in the bulk aerodynamic method. For the first time, the model is tested against observations that cover a wide range of aerodynamic roughness lengths $\left(z_{0}\right)$. During the experiments, performed in the ablation areas of the Greenland ice sheet and the Vatnajökull ice cap in Iceland, the surface varied from smooth snow to very rough hummocky ice. Over relatively smooth snow and ice with $z_{0}$ below a threshold value of approximately $10^{-3} \mathrm{~m}$, the model performs well and in accord with earlier studies. However, with growing hummock size, $z_{0}$ increases well above the threshold and the bulk aerodynamic flux becomes significantly smaller than the eddy-correlation flux (e.g. for $z_{0}=0.01 \mathrm{~m}$, the bulk aerodynamic flux is about $50 \%$ smaller). Apparently, the model severely underpredicts $z_{s}$ over hummocky ice. We argue that the surface renewal model does not account for the deep inhomogeneous roughness sublayer (RSL) that is generated by the hummocks. As a consequence, the homogeneous substrate ice grain cover becomes more efficiently 'ventilated'. Calculations with an alternative model that includes the RSL and was adapted for use over hummocky ice, qualitatively confirms our observations. We suggest that, whenever exceedance of the threshold occurs $\left(z_{0}>10^{-3} \mathrm{~m}\right.$, i.e., an ice surface covered with at least $0.3-\mathrm{m}$ high hummocks), the following relation should be used to calculate scalar roughness lengths, $\ln \left(z_{s} / z_{0}\right)=1.5-0.2 \ln \left(R e_{*}\right)-0.11\left(\ln \left(R e_{*}\right)\right)^{2}$.
\end{abstract}

Keywords Ablation area - Aerodynamic roughness length - Bulk aerodynamic flux . Ice surface roughness $\cdot$ Scalar roughness length $\cdot$ Surface renewal model

C. J. P. P. Smeets $(\varangle) \cdot$ M. R. van den Broeke

Institute for Marine and Atmospheric Research Utrecht, Princetonplein 5, 3584 CC, Utrecht,

The Netherlands

e-mail: c.j.p.p.smeets@uu.nl 


\section{Introduction}

For studying the response of ice caps or ice sheets to climate change the so-called ablation zone, where the yearly net surface ice melt takes place, is of particular importance. Investigation of the net surface energy balance in this area requires, amongst other things, reliable parametrisation of the surface exchange of momentum, heat and moisture. In mesoscale or large-scale atmospheric mumerical models, and for analysing automatic weather station (AWS) data, the robust bulk aerodynamic method is often used to calculate the turbulent surface flux. The key input parameters for this method are the aerodynamic and scalar roughness lengths, i.e., $z_{0}$ and $z_{s}$, respectively. Very little is known about their values and variations throughout the ablation area since micro-meteorological observations are scarce.

Although most studies adopt a constant value for $z_{0}$ in space and time, recent results in Smeets and Van den Broeke (2008) show that the spatial and temporal variations of $z_{0}$ in the lower ablation area of the Greenland ice sheet are very large (a factor 500). A comparable seasonal variability of $z_{0}$ was found by Smeets et al. (1999) in the ablation area of a broad outlet glacier from the Vatnajökull ice cap in Iceland during summer melt. These results demonstrate the large variability of $z_{0}$ in ablation areas and point out that rough hummocky ice can become the dominant surface cover in an ablation area during summer melt.

While the parametrisation of $z_{0}$ over ice surfaces is very complex, $z_{s}$ over ice and snow is often estimated using the surface renewal model presented by Andreas (1987). Up to now, the model of Andreas has been succesfully tested for relatively smooth snow and ice surfaces (Andreas 2002). In this paper, the Andreas model is tested for the first time, over a very wide range of surface roughness varying from smooth snow to very rough hummocky ice, by using the data from the experiments presented by SB08 and SDV99.

\section{Field Area, Instrumentation, and Data Selection}

The field area and instrumentation during the experiments in Greenland and Iceland are described extensively in SB08 and SDV99, respectively. Therefore we will only give a brief description here.

\subsection{Greenland}

The data were collected on the west side of the Greenland ice sheet at a location called S6 ( $38 \mathrm{~km}$ from the ice edge, 1,000 m.a.sl., slope $1^{\circ}$ ) in the period August 2003 to August 2004 and contain AWS and eddy-correlation measurements. The location is part of the so-called K-transect that is operated by the Institute for Marine and Atmospheric Research Utrecht (IMAU) (Van de Wal et al. 2005, SB08). The transect has been operated since 1990 and currently constitutes eight locations with mass balance and GPS measurements and three AWS. At the K-transect, the ice-sheet consists of a broad and sloping ablation area that extends from the ice edge, at $200 \mathrm{~m}$.a.s.1., about $90 \mathrm{~km}$ eastward to meet the equilibrium line around 1,500 m.a.s.l. Throughout the year, the boundary layer is dominated by a persistent katabatic flow that implies year-round stable stratification close to the surface and a wind speed maximum at some distance above (Van den Broeke et al. 1994, SB08).

The profile measurements at the AWS are performed at approximately 2-m and 6-m heights using R.M. Young 05103-L wind monitors for wind speed and direction, and radiation shielded non-ventilated temperature and humidity probes (Vaisala HMP35AC). A Kipp and Zonen CNR1 net radiometer is fixed at 6-m height, measuring all four radiation 
components separately, as well as the body temperature. On a separate mast, at a distance of 30-m from the AWS, a Campbell SR50 sonic height ranger is mounted. A Campbell CR10X datalogger, sampling every $30 \mathrm{~s}$, stored 30 -min averages.

Eddy-correlation measurements were made on a separate mast at 2.75-m height with a Campbell CSAT3 sonic anemometer, a Campbell FW3 Type E thermocouple, and a LI-COR LI7500 hygrometer. The correct operation of the hygrometer is limited to temperatures above $-25^{\circ} \mathrm{C}$. Every $10 \mathrm{~min}$ a value for the mean, standard deviation and covariance was calculated and stored on a Campbell CR23X datalogger. The raw data output rate of the eddy-correlation system was $10 \mathrm{~Hz}$.

\subsection{Iceland}

The experiments in Iceland were performed at the Vatnajökull ice cap as part of the Vatnajökull experiment (Oerlemans et al. 1999). The measurements presented are from two locations in the ablation area of the Breidamerkurjökull outlet glacier that is located at the south side of the ice cap, a few kilometer from the Atlantic coastline. The locations, referred to as A4 and A5, were situated at an altitude of 279 and $381 \mathrm{~m}$, the slope was $4^{\circ}$ and $2.5^{\circ}$, and the distance from the ice edge 1.2 and $5.4 \mathrm{~km}$, respectively. The experiments were carried out from 20 May to 31 August 1996 when the boundary layer was dominated by a persistent katabatic flow, implying stable stratification close to the surface and a wind maximum at some distance above. Both sites had the same configuration consisting of separate profile, turbulence, and radiation masts. The masts were positioned on a line perpendicular to the prevailing wind direction and about $20 \mathrm{~m}$ apart. The profile mast contained wind speed sensors (Vector Instruments, A100R) and radiation shielded and ventilated temperature and humidity probes (Vaisala HMP35AC) at five heights (approximately 1, 2, 3, 6, and 9 m), with a wind vane (Vector Instruments, W200P) placed at the top. The eddy-correlation system (at about 3-m height) consisted of a three-axis (axi-symmetric) research ultrasonic anemometer (Solent 1012 R2, Gill Instruments), two thermocouples (home manufactured), and a Lyman- $\alpha$ hygrometer (home manufactured). The radiation measurements were performed at about 4-m height with a shortwave radiometer (incoming and outgoing, Kipp pyranometer, CM7), two longwave radiometers (incoming and outgoing, Eppley pyrgeometer, model PIR), and one ventilated net radiometer (Schulze-Däke, Dr. Bruno Lange GMBH). Data collection was done with a Campbell $21 \mathrm{X}$ data logger, calculating values for the mean, standard deviation and covariance every $30 \mathrm{~min}$ and using a 5-min running mean to discriminate for low frequency contributions. The raw data output rate of the eddy-correlation system was $20 \mathrm{~Hz}$.

\section{The Bulk Aerodynamic Method}

\subsection{Background Theory}

The bulk aerodynamic method uses the difference in wind speed or scalar values between the surface and the observation height, via a transfer coefficient, to calculate the turbulence flux. For sensible heat this is written as follows

$$
H=\rho c_{p} C_{H z} u(z)\left[T_{0}-T(z)\right]
$$

and, 


$$
C_{H z}=\frac{k^{2}}{\left[\ln \left(z / z_{0}\right)-\alpha z / L\right]\left[\ln \left(z / z_{s}\right)-\alpha z / L\right]}
$$

where $H$ is the sensible heat flux, $\rho$ the air density, $C_{p}$ the specific heat of air, $C_{H z}$ the transfer coefficient, $u$ the wind speed, $T$ the temperature, $T_{0}$ the surface temperature, $k$ the von Kármán constant (0.40), $\alpha_{m}$ is a coefficient of the stability function taken as $5, L$ is the Obukhov length defined as $L=-u_{*}^{3} \theta_{v} / k g \overline{w \theta_{v}}$ where $g$ is the gravitational acceleration, $\theta_{v}$ the virtual potential temperature, and $\overline{w \theta_{v}}$ the virtual heat or buoyancy flux. For the calculation of $z / L$ from the profile data we use the same empirical relation as in SB08 (Pandolfo 1966)

$$
z / L_{P}=R i_{g} /\left(1-5 R i_{g}\right)
$$

with

$$
R i_{g}=\frac{g}{T} \frac{(\partial z / \partial T)}{(\partial u / \partial z)^{2}} .
$$

The key parameters for the calculation of the bulk aerodynamic flux are $z_{0}$ and $z_{s}$. In the following we discuss the methods that were used to derive their values.

\subsection{Aerodynamic Roughness Lengths}

In Fig. 1 we summarize the results from SDV99 and SB08 concerning the magnitude and variability of $z_{0}$. The curves are linear interpolations between time bin-averaged values of $z_{0}$. The year-round Greenland data show a large seasonal variation that matches the onset of the accumulation and ablation season. In August, at the end of the ablation season the surface is covered with hummocks and wide gullies and $z_{0}$ is large $\left(z_{0}>10^{-2} \mathrm{~m}\right)$. During winter, snow covers all surface irregularities and bridges the gullies so that $z_{0}$ becomes small $\left(z_{0} \approx 10^{-4} \mathrm{~m}\right)$. The Iceland data show the same rapid increase during the melt season as for the Greenland data. The surface at A4 and S6 is comparable and characterised by relatively smooth hummocks up to about $1 \mathrm{~m}$ height. At A5, $z_{0}$ becomes much larger since the

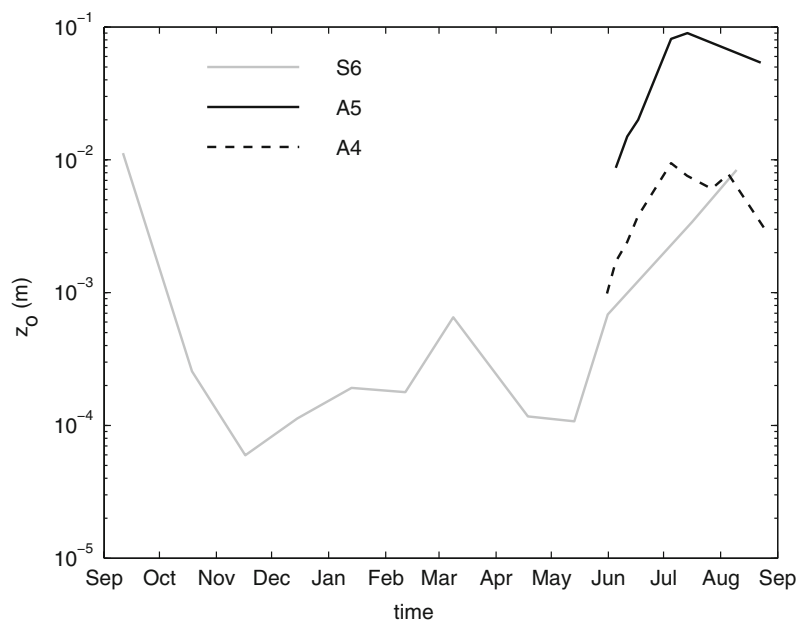

Fig. 1 The variation of aerodynamic roughness length as a function of time for location S6 in Greenland, and locations A4 and A5 in Iceland. The data are the median values of binned $z_{0}$ presented by SB08 and SDV99 
hummocks were higher ( $1.7 \mathrm{~m}$ on average), more ruggedly shaped and had a higher packing density. At the end of the melt season, $z_{0}$ at A4 and A5 decreases because the high melt rates eroded the large hummocks into slender and less voluminous obstacles (SDV99).

For the calculation of bulk aerodynamic fluxes we use the individual 30-min $z_{0}$ values derived from the eddy-correlation measurements to account for the strong time dependence of $z_{0}$. SB08 show that individual snow and melt events can rapidly change $z_{0}$ in Greenland. For Iceland data, this additionally rules out the effects of a wind directional dependency. The results are quality checked by including the criterion $0.9<u_{*} / u_{* b u l k}<1.1$ where $u_{*}$ and $u_{* b u l k}$ are the eddy-correlation and bulk aerodynamically-derived friction velocities.

\subsection{Scalar Roughness Lengths}

The scalar roughness length, $z_{s}$, is an essentially different parameter to $z_{0}$. The transfer of momentum is strongly affected by pressure fluctuations in turbulent wakes behind roughness elements (i.e. form drag) while the transfer of scalars depends ultimately on the molecular diffusion across the very thin viscous sublayer overlaying the surface (Garratt 1992). From considering the structure of the viscous sublayer, and using simple similarity arguments, a measure can be made of the relative values of $z_{0}$ and $z_{s}$ (Brutsaert 1975; Andreas 1987). The surface renewal model of Andreas (1987), our focus in this paper, is often used over snow and ice surfaces to calculate $z_{s}$. The results of the model are summarized in an equation that relates $z_{s}$ with the Reynolds roughness number, $R e_{*}=u_{*} z_{0} / v$ with $v$ the kinematic viscosity of air

$$
\ln \left(z_{s} / z_{0}\right)=b_{0}+b_{1} \ln \left(R e_{*}\right)+b_{2}\left(\ln \left(R e_{*}\right)\right)^{2}
$$

where $b_{0,1,2}$ are the polynomial coefficients (see Table 1). Most of our measurements were obtained in the rough flow regime and, since the coefficients for humidity and temperature are very close, we only apply the latter to calculate bulk aerodynamic fluxes.

When deriving scalar roughness lengths from eddy-correlation data, the surface temperature $T_{0}$ is often the most critical parameter. The measurements in Iceland were conducted during summer over a continuously melting ice surface so that $T_{0}$ was fixed. In Greenland, however, the data cover a whole year, and surface melt only occured during the daytime hours in summer. For this location, we estimated $T_{0}$ from the longwave outgoing radiation. Our measurements over melting ice, however, show biased values of $T_{0}$ ranging between +0.5 and $+1.0^{\circ} \mathrm{C}$. It is well-known that the output of a longwave radiation sensor output is subject to several corrections that relate to the influence of incoming shortwave radiation (Fairall et al. 1998). For cases with no shortwave radiation and a very small temperature gradient between 2-m and 6-m heights $\left(|\Delta T|<0.05^{\circ} \mathrm{C}\right)$, we find that the temperature difference between $2 \mathrm{~m}$ and the surface is less than $\pm 0.3^{\circ} \mathrm{C}$ for all data with $T<0^{\circ} \mathrm{C}$. This result implies a correct calibration of the instrument and we therefore assume that the biased results relate to the

Table 1 Values for the polynomial coefficients in Eq. 5 for three different aerodynamic flow regimes (Andreas 1987)

\begin{tabular}{llll}
\hline & $\begin{array}{l}R e_{*} \leq 0.135 \\
\text { smooth flow }\end{array}$ & $\begin{array}{l}0.135<R e_{*}<2.5 \\
\text { transitional }\end{array}$ & $\begin{array}{l}2.5 \leq R e_{*} \leq 1000 \\
\text { rough flow }\end{array}$ \\
\hline$b_{0}$ & 1.25 & 0.149 & 0.317 \\
$b_{1}$ & & -0.550 & -0.565 \\
$b_{2}$ & & & -0.183 \\
\hline
\end{tabular}


effects of incoming shortwave radiation. We related the bias, for cases with a melting ice surface, to the sum of the shortwave radiation components and assume that this correction is valid over the whole temperature range.

\section{Data Corrections and Selection Criteria}

The eddy-correlation systems in Greenland and Iceland were subject to imperfect levelling due to the surface slope and variable summer melt. The latter prevents the use of a fixed rotation, i.e. the planar fit method (Wilczak et al. 2001), and therefore we force the individual half-hourly averages of $\bar{v}$ and $\bar{w}$ to zero. In the case of a stationary and homogeneous boundary layer in a two-dimensional and axially symmetric flow, $\overline{v^{\prime} w^{\prime}}$ should now equal zero, but in practice this is seldom the case. As part of the data selection criteria we therefore set $\left|\overline{v^{\prime} w^{\prime}} / \overline{u^{\prime} w^{\prime}}\right|<0.25$. All eddy-covariance data were corrected for pathlength averaging between the sensor heads and sensor separation (Moore 1986). Corrections to the sonic temperature flux involve the contamination from humidity and momentum fluxes (Schotanus et al. 1983). The effect of density fluctuations on the water vapour flux (WPL corrections, after Webb et al. 1980) is very small (i.e. between +1 and $-2 \%$ ) and neglected because it lies well within the range of measurement accuracy. The persistent katabatic flow at both locations ensures narrow wind sectors as given in SB08 and SDV99.

The remaining selection criteria are $0<z / L<0.2, u>4 \mathrm{~ms}^{-1}, u_{*}>0.1 \mathrm{~ms}^{-1}$, $-300<H<-20 \mathrm{~W} \mathrm{~m}^{-2}$, and $-50<L E<-10 \mathrm{~W} \mathrm{~m}^{-2}$ where $L E$ is the latent turbulent heat flux. Joffre (1982) shows that the stationarity of runs is most effectively evaluated from temperature variations and we demand that the temperature change between consecutive half-hourly runs is less than $0.3^{\circ} \mathrm{C}$. We do not analyse the latent heat fluxes from the Iceland experiment because these were too small.

\section{Data Analysis}

\subsection{Eddy-Correlation and Bulk Aerodynamic Fluxes}

The performance of the Andreas model is studied over a wide range of ice surface roughness by plotting the ratio of the eddy-correlation and bulk aerodynamic fluxes as a function of (binned) $z_{0}$ values (Fig. 2). The number of half-hourly values contained in all bins is 1689 , 338, 424, and 266 for Greenland sensible heat, latent heat, and Iceland sensible heat fluxes from A4 and A5, respectively. Bins containing less than five values are not plotted. The size of the error bar equals twice the average standard deviation of all bins.

Below a threshold value of about $z_{0} \approx 10^{-3} \mathrm{~m}$ the fluxes are about equal, which suggests that the model of Andreas correctly predicts $z_{s}$. In the review by Andreas (2002) and the study by Denby and Snellen (2002) the predictions from the Andreas model are also supported. It is striking that for all earlier experiments $z_{0}$ did not exceed the threshold value. Furthermore, all experiments with $z_{0}$ close to the threshold value (Munro 1989; Smeets et al. 1998; Denby and Snellen 2002) were performed over relatively smooth melting glacier ice with a homogeneous cover of ice grains. Above the threshold, the ratio of the fluxes increases well above 1 so that $z_{s}$ is seriously underestimated by the model of Andreas. Despite the differences in location, conditions, instrumental set-up, and scalars studied, this divergence from the model predictions is observed in a consistent fashion for all available data. This 
Fig. 2 Ratio of eddy-correlation (EC flux) and bulk aerodynamic fluxes (bulk flux) plotted as a function of $z_{0}$. Black circles and crosses are the sensible and latent heat fluxes, respectively from the Greenland experiment. Grey triangles and squares are the sensible heat fluxes from A4 and A5, respectively, from the Iceland experiment. The error bar on the left equals two times the average standard deviation of all bins

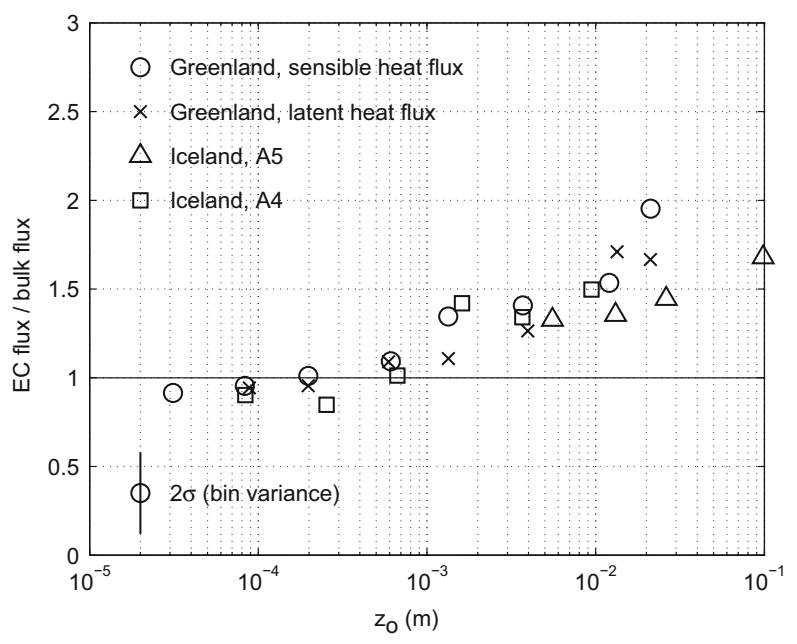

indicates an intimate link between the threshold, the microscale roughness (ice grains) and scalar transfer as modelled by Andreas (1987).

The threshold value is also clearly linked to the observations from SB08 who find that the total surface drag is dominated either by the microscale surface roughness (ice grains) below it or by hummocks above it. Apparently, hummocks efficiently increase both the total surface drag and the scalar transfer efficiency (discussed in Sect. 6). It is unlikely that the threshold has a single universal value and it probably varies with the characteristics of the microscale roughness around $10^{-3} \mathrm{~m}$. From Fig. 2 we estimate that for our experiments the threshold value is $z_{0}=7 \times 10^{-4} \mathrm{~m}$.

\subsection{Scalar Roughness Lengths}

In Fig. 3, we present our results for $z_{s}$ in the same way as was done by Andreas (1987) to present his model results, i.e. non-dimensionalised with $z_{0}$ and plotted as a function of $R e_{*}$. The results from the model are displayed as a solid grey line. For a better identification of possible trends in the various data sets, we averaged our data in equally sized $R e_{*}$ bins by taking the logarithm of the individual $z_{s} / z_{0}$ ratios. The size of the error bar equals twice the average standard deviation of all bins. Following the results in the previous section, we split the data into two classes using the threshold value $z_{0}=7 \times 10^{-4} \mathrm{~m}$. Runs with $z_{0}$ below and above the threshold value are plotted as black and grey symbols, respectively. The results from Smeets et al. (1998) are included to serve as a reference to data with $z_{0}$ close to the threshold value (black dots); the grey area contains data with $z_{0}$ close to the threshold value, and is bounded by $R e_{*}$ values using $z_{0}=7 \times 10^{-4} \mathrm{~m}$ and $0.1<u_{*}<0.9 \mathrm{~m} \mathrm{~s}^{-1}$.

In accord with earlier findings, data obtained below the threshold follow the Andreas curve. With $z_{0}$ above the threshold, all data lie systematically higher and parallel to the Andreas curve. The overlap of both datasets in the threshold region (grey area) indicates how sudden the scalar transfer efficiency increases when going from smooth to hummocky ice. Moreover, this overlap prevents the calculation of a new curve that replaces the Andreas model in the aerodynamically rough flow regime. Such a curve would inevitably leed to large errors in the calculation of $z_{s}$ in the region of overlap. Instead, the results from Andreas in the aerodynamically rough flow regime are extended with a second curve (solid black line) 


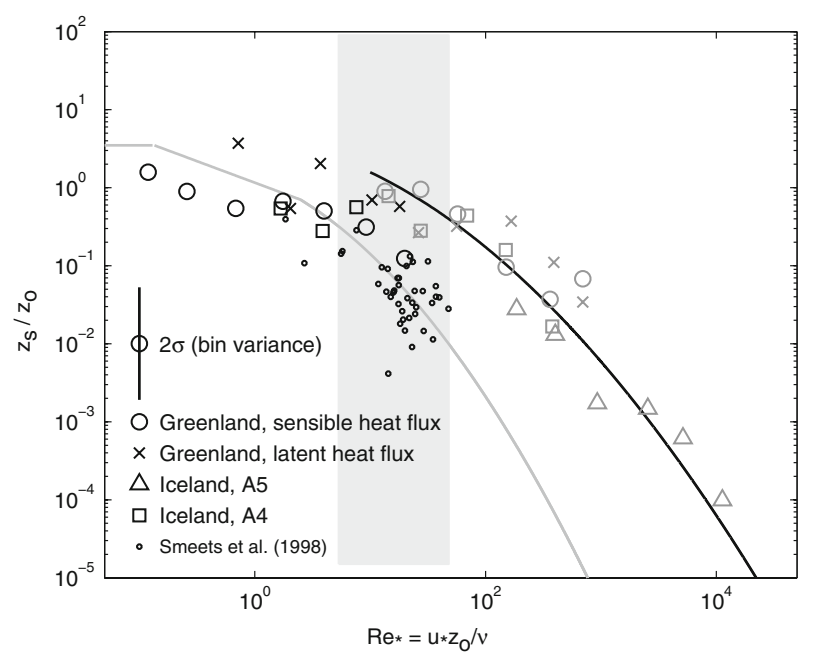

Fig. 3 The ratio of scalar and aerodynamic roughness lengths plotted as a function of $R e_{*}$. The smooth grey and black solid line are the prediction from the Andreas model and the fit to our hummocky ice data, respectively. The grey area indicates the threshold region with $z_{0}=7 \times 10^{-4} \mathrm{~m}$ and $0.1<u_{*}<0.9 \mathrm{~ms}^{-1}$. The black and grey symbols represent data obtained below and above the threshold value, respectively. The error bar on the left equals two times the average standard deviation of all bins. The data from Smeets et al. (1998) are used as a reference to a smooth melting ice surface

Table 2 Values for the polynomial coefficients in Eq. 5 for the case of aerodynamically rough flow, i.e., $R e_{*}>2.5$

\begin{tabular}{lll}
\hline & Smooth ice $z_{0}<10^{-3} \mathrm{~m}$ & Hummocky ice $z_{0}>10^{-3} \mathrm{~m}$ \\
\hline$b_{0}$ & 0.317 & 1.5 \\
$b_{1}$ & -0.565 & -0.2 \\
$b_{2}$ & -0.183 & -0.11
\end{tabular}

The smooth ice results are from Andreas (1987) and the hummocky ice result is estimated from our hummocky ice data. The use of the smooth or hummocky ice coefficients is determined by the threshold value $z_{0} \approx 10^{-3} \mathrm{~m}$

that applies whenever $z_{0}>10^{-3} \mathrm{~m}$. The coefficients are listed in Table 2 together with those from Andreas. We estimate from drag model calculations (SB08) and field observations that a homogeneous cover with hummocks higher than about $0.3 \mathrm{~m}$ approximates surface conditions with $z_{0}>10^{-3} \mathrm{~m}$.

In Fig. $4, z_{s}$ is plotted as a function of $z_{0}$ using logarithmically averaged and binned values. The results from the model and our hummocky ice curve are plotted as grey areas A and B, respectively, using a range of conditions with $0.1<u_{*}<0.9 \mathrm{~m} \mathrm{~s}^{-1}$. Below the threshold value, the data compare to earlier findings (area A), while above the threshold, $z_{s}$ increases at the same rate as $z_{0}$ and attains unusually high values around $10^{-3} \mathrm{~m}$ (area B). At A5, $z_{s}$ has systematically lower values than at S6 while A4 varies in between. The larger hummocks, higher packing density and larger ice grains at A5 result in less effective scalar transfer as will be explained in the Sect. 6 and the Appendix.

The influence of drifting and blowing snow on $z_{s}$ was studied by Bintanja and Reijmer (2001) and Andreas et al. (2005). Bintanja and Reijmer (2001) found constant $z_{s}$ 


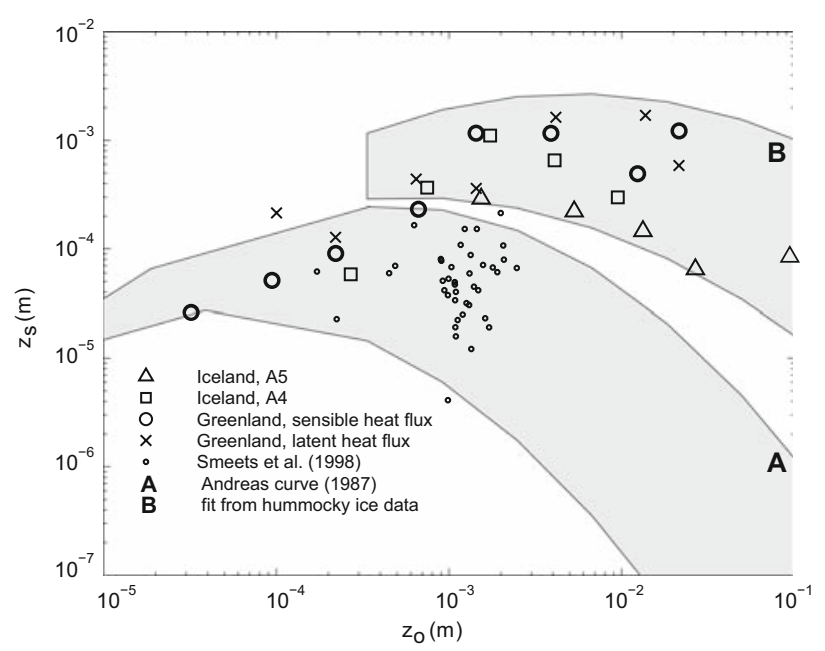

Fig. 4 Scalar versus the aerodynamic roughness lengths. The upper (A) and lower (B) grey area represent the envelope of results from our rough ice data fit and the Andreas model, respectively within the data range $0.1<u_{*}<0.9 \mathrm{~m} \mathrm{~s}^{-1}$. The data from Smeets et al. (1998) represent a reference to a smooth melting glacier ice surface with $z_{0} \approx 10^{-3} \mathrm{~m}$

Fig. 5 Scalar and aerodynamic roughness lengths versus the friction velocity for wintertime Greenland data. The thin and thick error bars equal two times the average standard deviation in a bin for scalar and aerodynamic roughness lengths, respectively

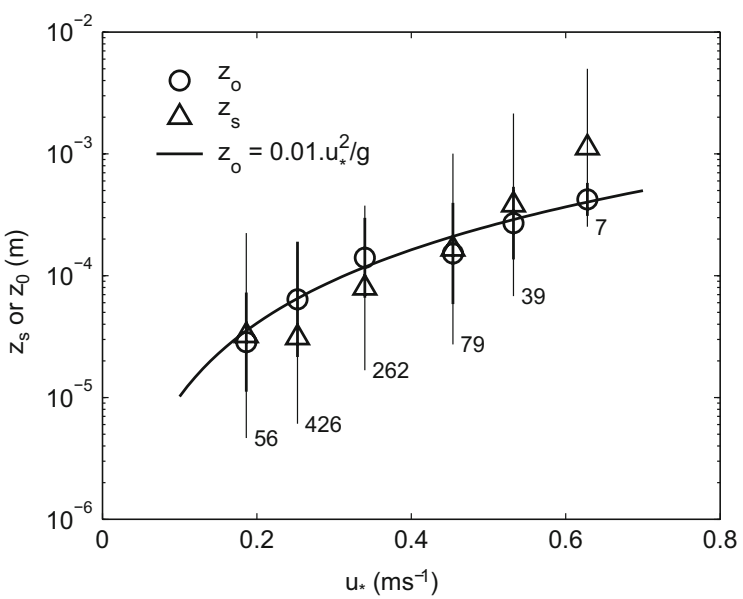

(i.e. $2 \times 10^{-4} \mathrm{~m}$ ) when $u_{*}$ was below the drifting snow threshold of $0.3 \mathrm{~m} \mathrm{~s}^{-1}$ and an increase as high powers of $u_{*}$ above this value. In contrast, Andreas et al. (2005) found no evidence for a variation with $u_{*}$. In Fig. 5, we reproduce the results from SB08 for $z_{0}$ as a function of $u_{*}$ and include, for the same selection of data, $z_{s}$. As argued in SB08, $z_{0}$ increases with $u_{*}$ due to snow particles taken up in the air (the power law increase of $z_{0}$ in a flow with a saltation layer, $z_{0}=0.01 u_{*}^{2} / g$, Owen 1964). We find that $z_{s}$ increases at the same rate as $z_{0}$ with error bars that are about twice as large. 


\section{Discussion}

In the model of Andreas, the atmospheric surface layer is represented by a thin viscous sublayer overlying the surface and a semi-logarithmic inertial sublayer on top of that (left drawing, Fig. 6). Within the former layer, molecular diffusion dominates the transfer of scalars while in the latter layer similarity theory is valid. Andreas (1987) derives scalar profiles in the viscous sublayer by using the surface renewal concept and matches these with the inertial sublayer profiles yielding estimates for the scalar roughness lengths.

The surface renewal concept was suggested by Danckwerts (1970) and confirmed by the observations of Grass (1971). Small eddies that are in contact with the surface may become stagnant as they are trapped for a short period of time between the roughness elements. In other words, we can think of the viscous sublayer as a layer consisting of small eddies being continually replaced. Since the eddies are presumed to be periodically stagnant, the transfer process between these eddies and the surface can be described by a diffusion process (Brutsaert 1975; Andreas 1987). The efficiency of the transfer process across the viscous sublayer is related to the roughness Reynolds number $R e_{*}=u_{*} z_{0} / \nu$.

With $z_{0}$ below the threshold, the model of Andreas performs well, indicating that the inertial sublayer scales $z_{0}$ and $u_{*}$ properly scale the scalar transfer efficiency close to the microscale roughness (left drawing, Fig. 6). Above the threshold, hummocks dominate the total surface drag (SB08) and the flow close to the surface becomes very inhomogeneous depending on the location, orientation, and dimension of the hummocks (Raupach et al. 1980). The model of Andreas (1987) does not account for the effects of a RSL and it is easy to imagine that, when the viscous sublayer is embedded in the inhomogeneous RSL (right drawing, Fig. 6), local RSL scales are more appropriate than those derived from inertial sublayer characteristics (i.e., $z_{0}$ and $u_{*}$ ).

The scalar transfer process over hummocky ice is qualitatively studied with the model of Brutsaert (1979) developed for surfaces covered with uniform and dense (isothermal) vegetation. In this model, viscous sublayer transfer takes place within the plant canopy (i.e., representative of a RSL) at the scale of the individual leaves, and consistent with that, the length and velocity scales relate to the leaf size and an exponentially decaying canopy profile of $u_{*}$, respectively. In the case of a hummocky ice surface, the leaves and the canopy height resemble the ice grains and the hummock height, respectively. The model of Brutsaert

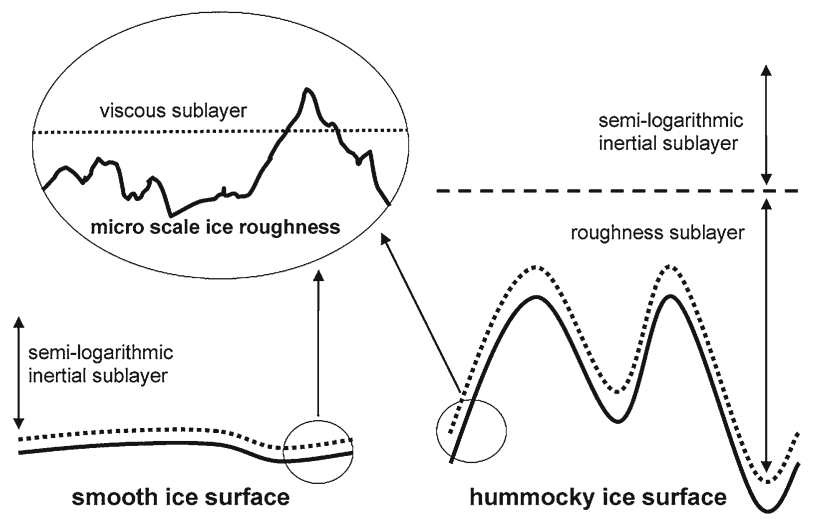

Fig. 6 Schematic representation of a smooth and hummocky ice surface covered homogeneously with microscale roughness 
(1979) and the modifications for use over hummocky ice are described in the Appendix. The model results qualitatively agree with our observations and resolve the differences observed between A5 and S6. Application of the local scales and transport properties present in the RSL indeed result in a substantially higher scalar transfer efficiency than found over a smooth ice or snow surface.

As also suggested for the Andreas model (Andreas 2002), the application of the hummocky ice curve (Table 2) is not necessarily specific to ice. The curve should in general apply to bare surfaces covered with large enough bluff roughness elements to exceed a certain threshold for $z_{0}$. For these cases, the total surface drag is dominated by the hummocks and a well-developed RSL is present. The general application of our results is confirmed by a recent study of Kanda et al. (2007) who present a remarkably similar curve for heat transfer over outdoor urban scale models. Furthermore, recent observations over various bare-soil surfaces in China by Yang et al. (2008) also agree, to some extent, with both curves.

\section{Conclusions}

The surface renewal model of Andreas (1987) is widely used over snow and ice surfaces to estimate the scalar roughness length $\left(z_{s}\right)$, a key parameter in the calculation of bulk aerodynamic fluxes. Up to now, the model predictions have been verified over relatively smooth snow and ice surfaces (Andreas 2002; Denby and Snellen 2002). However, as is shown by Smeets et al. (1999, referred to as SDV99) and Smeets and Van den Broeke (2008, referred to as SB08), rough hummocky ice can be the dominant surface cover in ablation areas during summer melt. We tested, for the first time, the model of Andreas (1987) over a very wide range of snow and ice surface roughnesses. The data studied are year-round observations from a location in the ablation area of the Greenland ice sheet (SB08) and summertime observations from two locations at the Vatnajökull ice cap in Iceland (SDV99). The range of surface conditions during the experiments varied between smooth snow or ice and very rough hummocky ice $\left(10^{-4}<z_{0}<10^{-1} \mathrm{~m}\right)$.

Over relatively smooth snow and ice, with $z_{0}$ below a threshold value of $10^{-3} \mathrm{~m}$, the Andreas model performs well and in accord with earlier studies (Andreas 2002; Denby and Snellen 2002). However, when during summer melt the ice surface becomes hummocky, $z_{0}$ increases above the threshold value and the bulk aerodynamic flux becomes increasingly smaller than the eddy-correlation flux (e.g. for $z_{0}=0.01 \mathrm{~m}$ the bulk flux is about $50 \%$ smaller). Consequently, the model of Andreas (1987) significantly underestimates $z_{s}$ over hummocky ice.

In the model of Andreas (1987), scalar transfer is described by matching the viscous and inertial sublayer profiles. In the case of hummocky ice, the viscous sublayer becomes embedded in a highly inhomogeneous roughness sublayer (RSL), which is not accounted for in the model. Local scales and transport properties within the RSL determine the efficiency of scalar transfer. We adapted a model, originally developed for use over vegetated surfaces (Brutsaert 1979), for application over hummocky ice. The model results qualitatively agree with our observations and support the idea that the RSL promotes more efficient 'ventilation' of the substrate ice grain cover.

We extend the results of Andreas (1987) for aerodynamically rough flow with a second set of coefficients that should be applied whenever the threshold $z_{0} \approx 10^{-3} \mathrm{~m}$ is exceeded, i.e., $\ln \left(z_{s} / z_{0}\right)=1.5-0.2 \ln \left(R e_{*}\right)-0.11\left(\ln \left(R e_{*}\right)\right)^{2}$. From drag model calculations and field observations we estimate that the threshold value corresponds to an ice surface covered with about $0.3 \mathrm{~m}$ high hummocks. Moreover, the latter curve probably applies in general to all 
bare surfaces covered with sufficiently large bluff roughness elements. Kanda et al. (2007) find a remarkably similar curve in a study of heat transfer over outdoor urban scale models and the observations from Yang et al. (2008) over various bare-soil surfaces also agree, to some extent, with our curve.

Acknowledgements We would like to thank the IMAU technicians for designing and maintaining the AWS and turbulence stations, and all the people involved in the data collection. Furthermore, the comments from two anonymous referees are very much appreciated, as is the helpful discussion with Kun Yang from whom we obtained the two recent references on observations over outdoor urban scale models and bare-soil surfaces. This work is funded by the Utrecht University and the Netherlands Polar Program (NPP) of the Netherlands Organisation of Scientific Research, section Earth and Life Sciences (NWO/ALW).

Open Access This article is distributed under the terms of the Creative Commons Attribution Noncommercial License which permits any noncommercial use, distribution, and reproduction in any medium, provided the original author(s) and source are credited.

\section{Appendix: A Model Study of Scalar Transfer over Rough Ice}

The transfer model that we use to study scalar transfer over rough ice is described extensively in Brutsaert (1979) and Brutsaert (1982). A comparable model was also presented by Shreffler (1976). The model is originally used to describe the transport of a scalar admixture of the flow to and from a surface covered with uniform and dense vegetation, or similar fibrous roughness elements. Likewise, in the model of Andreas (1987), a surface renewal concept is used to describe the transfer across the viscous sublayer and the model also includes a transitional layer between the viscous sublayer and the inertial sublayer. The concept of the model agrees with our ideas of transport over hummocky ice as discussed in Sect. 6.

Brutsaert (1979) uses simple similarity arguments to describe the turbulent flow in plant canopies. The resulting differential equation is solved for the specific case of an isothermal foliage surface to yield expressions for the transfer coefficients. In reality, a vegetational surface is mostly anisothermal and Brutsaert and Sugita (1996) extend the solution to this problem. A (melting) hummocky ice surface, however, is a good example of an isothermal surface.

We describe the model in plant canopy terms and between brackets we place the anology for a hummocky ice surface. Furthermore, the adaption of all the important parameters to a hummocky ice surface will be extensively discussed. Thereafter, the model is used to qualitatively study scalar transfer for location S6 in Greenland and A5 in Iceland since these have different surface and transfer characteristics (Sect. 5.2).

Turbulent transfer of any scalar is assumed to be governed by

$$
d F / d z-S_{f}=0
$$

with $F$ the vertical flux through the canopy (or RSL) and $S_{f}$ the source term emanating from the leaves (or ice grain). The flux-profile relationship can be expressed in terms of an eddy diffusivity

$$
K_{c}=\frac{-(F / \rho)}{d \bar{c} / d z)}
$$

with $\bar{c}$ the mean concentration of any physical quantity (for sensible heat this is e.g. $c=c_{p} \bar{T}$ ). For the following type of sollution it is required that the concentration at the foliage surface $c_{s}$ (or ice grain) is constant or that the surface is isothermal. Both criteria are sufficiently fullfilled for hummocky ice, even in the case when the surface is not melting. 
To solve the set of Eqs. 6 and 7, we assume that the shape of $K_{c}$ within the canopy (or between hummocks) can be described with the same exponential decaying function as is found for $\bar{u}, u_{*}$ or $K_{m}$ inside a wide range of vegetational canopies or sparse rigid elements. Thus, assuming Reynolds anology, the eddy diffusivity can be described as

$$
K=K(H) \exp (-a \xi)
$$

in which $\xi=(H-z) / H$ with $K(H)$ the eddy diffusivity at the top of the obstacle or canopy, $a$ the extinction parameter (assumed equal for all parameters), $H$ the height of the canopy (or hummock), and $z$ the height within the canopy (or between hummocks).

A value for $a$ over hummocky ice is derived from studying literature concerning bluff hummock type roughness. Macdonald (2000) shows that the exponential decaying function fits the profiles between in-line and staggered cube arrays in a wind tunnel as long as the packing density is not too high (i.e., $\lambda<0.3$, with $\lambda$ the ratio of frontal obstacle area divided by the unit surface area available for each obstacle). Moreover, Macdonald (2000) gives a linear relationship between $\lambda$ and $a$, i.e., $a \approx 10 \lambda$. For hummocky ice, $\lambda$ varies roughly between 0.05 and 0.15 (SDV99; SB08) so that we can use this relationship. Note, with respect to this, that hummocks are more aerodynamically shaped than cubes, and consequently $a$ is likely to be somewhat smaller than the predictions of Macdonald (2000).

The source term in Eq. 6 results from the flux from or to the foliage elements (or ice grain). It can be described with the following bulk transfer equation

$$
S_{f}=A C \rho u_{* c}\left(\bar{c}_{s}-\bar{c}\right)
$$

with $C$ the bulk transfer coefficient of the foliage elements (or ice grains), $u_{* c}$ the friction velocity close to the foliage elements (or ice grains), $c_{s}$ the concentration or temperature at the surface of the leaves (or ice grains) and $A$ the surface area (both sides) of leaves per unit volume of air. Parameter $A$ is an estimate of the total surface area of 'active' viscous sublayer per unit volume of the canopy. In the case of a hummocky ice surface, $A$ represents the exposed surface area of all ice grains within the volume made up of the unit surface area and height of a hummock. The ice grains are assumed to be shaped as a pyramid for which all sides are equal. In the case that they lie adjacent to each other, the maximum exposed pyramid area per unit ground area is 1.732 . The size of a pyramid side ranges from $0.005 \mathrm{~m}$ in Greenland to $0.03 \mathrm{~m}$ in Iceland. In addition, hummocks also increase the exposed surface area $(S I)$, and using the drag model from SB08, we estimated that $S I$ is about 1.15 and 1.35 for S6 and S5, respectively.

In reality, the exposed surface area will not be fully covered by an 'active' viscous sublayer for several reasons. Depending on the size of the pyramids/ice grains and turbulence intensity close to the surface, the pyramids can be either embedded, poke through, or be fully covered with a viscous sublayer in line with the surface renewal concept (see Sect. 6 and right drawing in Fig. 6). Moreover, the packing density of ice grains will be less than $100 \%$. We estimate this surface exposure reduction and its consequent uncertainties by applying two estimates for the reduction factor $C E$ in the model calculations (i.e. 0.75 and 0.5). Furthermore, the wake flow around hummocks causes sheltering of the surface, and hence a reduction in the area with an 'active' viscous sublayer. Using the drag model from SB08, we estimate that the sheltering efficiency $(\mathrm{SH})$ of hummocks is 10 and $20 \%$ for S6 and A5, respectively (i.e., $S H$ is 0.9 and 0.8 , respectively). The value for $A$ can now be calculated from $A=1.732 \times C E \times S H \times S I / H$. As is obvious from the above, the exact value of $A$ depends on so many parameters that it is difficult to estimate for any surface and it probably is responsible for the largest model uncertainty. The sensitivity of the results to uncertainties in $A$ follows from the variation of $C E$. 
From dimensional analysis it follows that the bulk transfer coefficient $C$ probably has the form

$$
C=C_{L} \operatorname{Re}_{* c}^{-m} \operatorname{Pr}^{-n}
$$

in which $R e_{* c}=u_{* c} L_{f} / v$ is a local Reynolds number, $L_{f}$ is a characteristic size of the foliage elements (or ice grains), and $\mathrm{Pr}$ is the Prandtl number. The parameters $C_{L}, m$ and $n$ may depend on shape, density and orientation of the foliage elements (or ice grains) and on the intensity of the turbulence. In his Appendix B, Brutsaert (1979) reviews past work and gives likely values for these parameters. As in Brutsaert (1975) and Brutsaert (1979), Andreas (1987) uses the surface renewal concept to describe viscous sublayer transfer. The Andreas model predictions are verified for cases below the threshold $z_{0} \approx 10^{-3} \mathrm{~m}$, hence, for cases where the microscale roughness determines the total drag. We therefore assume that Andreas's choice of parameters in Eq. 10 is suitable to describes the transfer process close to the ice grains, i.e., $C_{L}=0.18, m=0.25$, and $n=0.5$. Notice that Brutsaert (1979) uses $C_{L}=0.25$, a value derived from the experimental results of Chamberlain (1966) over grass-like roughness. Andreas (1987) derived his value for $C_{L}$ from a study by (Chamberlain 1968) over bluff roughness elements, which is more in line with ice grain roughness.

When $\bar{c}_{s}$ is constant it is convenient to normalize the concentration

$$
\chi=\frac{\bar{c}_{s}-\bar{c}}{\bar{c}_{s}-\bar{c}_{H}}
$$

where $\bar{c}_{h}$ is the concentration at $z=H$ and $H$ is the height of the canopy (or hummocks). Substitution of Eqs. 7, 9, 10 and 11 into 6 yields

$$
\frac{\mathrm{d}^{2} \chi}{\mathrm{d} \xi^{2}}+C_{1} \frac{\mathrm{d} \chi}{d \xi}-C_{2} e^{N \xi}=0
$$

in which $C_{1}=-a$,

$$
C_{2}=\frac{A C_{L} H^{2}}{k(H-d) \operatorname{Re}_{*}^{m} \operatorname{Pr}^{n}}
$$

and $N=[-a(1-m)+a]$ with $R e_{*}=u_{*} L_{f} / v$ and $u_{*}$ the friction velocity at $z=H$. To derive the bulk transfer coefficient, the upper boundary condition $z=H$ is taken as $\chi=1$, $\epsilon=0$. The lower boundary condition can vary from one problem to the other. Brutsaert (1979) discusses this problem and suggests that for cases with equal concentration and temperature between the foliage and ground surface the following condition can be used. Near the top of the canopy (or hummocks) the effect of the ground surface is not 'felt' so that the exact formulation of the condition at the ground is not important. This is in fact already implicit in the exponential profiles that are used to describe the flow parameters. The lower boundary condition now becomes $\chi=0, \xi \rightarrow$ large. The solution of Eq. 12, using these boundary conditions, allows calculation of the flux at $z=H$ by means of Eqs. 7 and 8

$$
F=-k[(H-d) / H] \rho u_{*}\left(\bar{c}_{s}-\bar{c}\right) G_{0}
$$

where $G_{0}=\mathrm{d} \chi / \mathrm{d} \xi$ is the gradient at $\xi=0$; this is

$$
G_{0}=-C_{2}^{1 / 2} K_{\lambda-1}\left(\frac{2 C_{2}^{1 / 2}}{m a}\right) / K_{\lambda}\left(\frac{2 C_{2}^{1 / 2}}{m a}\right)
$$

where $C_{2}$ is defined in Eq. 13 and where $K_{\lambda}(\mathrm{l})$ is the modified Bessel function of order $\lambda=m^{-1}$ of the second kind (Abramowitz and Stegun 1964). 
The surface sublayer profiles are commenly described with Monin-Obhukov similarity theory. If $c_{r}$ is the concentration at a reference level $z_{r}$ above the canopy (or hummocks), we can write for neutral conditions

$$
c_{H}-c_{r}=\frac{F}{k u_{*} \rho} \ln \left[\left(z_{r}-d\right) /(H-d)\right] .
$$

Elimination of $c_{H}$ between Eqs. 14 and 16 yields

$$
c_{s}-c_{r}=\frac{F}{k u_{*} \rho}\left\{\frac{H}{(H d)\left(-G_{0}\right)}+\ln \left[\left(z_{r}-d\right) /(H-d)\right]\right\} .
$$

Finally, the scalar roughness length, $z_{s}$, can be defined as follows,

$$
c_{s}-c_{r}=\frac{F}{k u_{*} \rho} \ln \left[\left(z_{r}-d\right) / z_{s}\right],
$$

and substituting in Eq. 17 yields

$$
\ln \left(z_{0} / z_{s}\right)=H /\left[(H-d)\left(-G_{0}\right)\right]-\ln \left[(H-d) / z_{0}\right] .
$$

All important parameter values that were used in the model calculations are summarized in Table 3. Part of these values were derived from studying the surface characteristics with the drag model presented in SB08 (i.e., $z_{0}, \lambda, S H, S I$ ).

In Fig. 7 we plotted the results from the model (grey areas) for the locations S6 in Greenland (A) and A5 in Iceland (B) together with the same data as in Fig. 3. At S6 and A5 the surfaces are distinctly different with at S6 (and also A4 in Iceland) relatively smooth and homogeneous hummocks while at A5 the hummocks were more rugged, higher and had a higher packing density. To address the sensitivity of the model results to uncertainties in $A$ we varied parameter $C E$ between 0.75 and 0.5 , which gives us the upper and lower boundary of the grey areas, respectively.

The model results are well in line with the data and also resolve the differences between S6 and A5. The sensitivity of the results to a $30 \%$ change of $A$ (by varying $C E$ from 0.75 to 0.5 ) is quite large, as follows from the vertical extent of the grey areas. When $C E$ decreases, the surface area of 'active' viscous sublayer reduces, and with that, the transport efficiency and $\ln \left(z_{s} / z_{0}\right)$. There are several reasons why the modelled ratios for A5 are smaller than those from S6. As $\lambda$, and with that, $a$ is larger at A5, the 'ventilation' between the hummocks is assumed to be lower. Increasing $\lambda$ for $S 6$ to the value of A5 (i.e., from 0.035 to 0.09), and

Table 3 List of site specific model parameters that were used to generate results for location S6 (Greenland) and A5 (Iceland)

\begin{tabular}{lll}
\hline Parameter & S6 & A5 \\
\hline$z_{0}$ aerodynamic roughness length $(\mathrm{m})$ & 0.01 & 0.04 \\
$\lambda$ frontal area index & 0.05 & 0.09 \\
$a$ profile extinction & 0.35 & 0.9 \\
$n / S$ unit surface area per hummock $\left(\mathrm{m}^{-2}\right)$ & $3 / 60$ & $2.5 / 100$ \\
$L_{f}$ characteristic size ice grain $(\mathrm{m})$ & 0.005 & 0.03 \\
$H$ hummock height $(\mathrm{m})$ & 0.5 & 1.7 \\
$S H$ shelter efficiency of hummocks & 0.9 & 0.8 \\
$S I$ surface area increase by hummocks & 1.15 & 1.35 \\
$C E$ 'active' sublayer coverage factor & 0.75 or 0.5 & 0.75 or 0.5 \\
$A$ exposed surface area per unit volume $\left(\mathrm{m}^{-1}\right)$ & 2.6 or 1.7 & 0.8 or 0.6
\end{tabular}


Fig. 7 The ratio of scalar and aerodynamic roughness lengths plotted as a function of $R e_{*}$. The smooth grey and black solid lines are the prediction from the Andreas model and the fit to our rough ice data, respectively. The grey area indicates the threshold region with $z_{0}=7 \times 10^{-4} \mathrm{~m}$ and $0.1<u_{*}<0.9 \mathrm{~ms}^{-1}$. The black and grey symbols represent data obtained below and above the threshold value, respectively. The two grey areas marked A and B are the results from the model for location S6 and A5. The upper and lower curve of each grey area was calculated by varying $E C$ from 0.75 to 0.5 , respectively

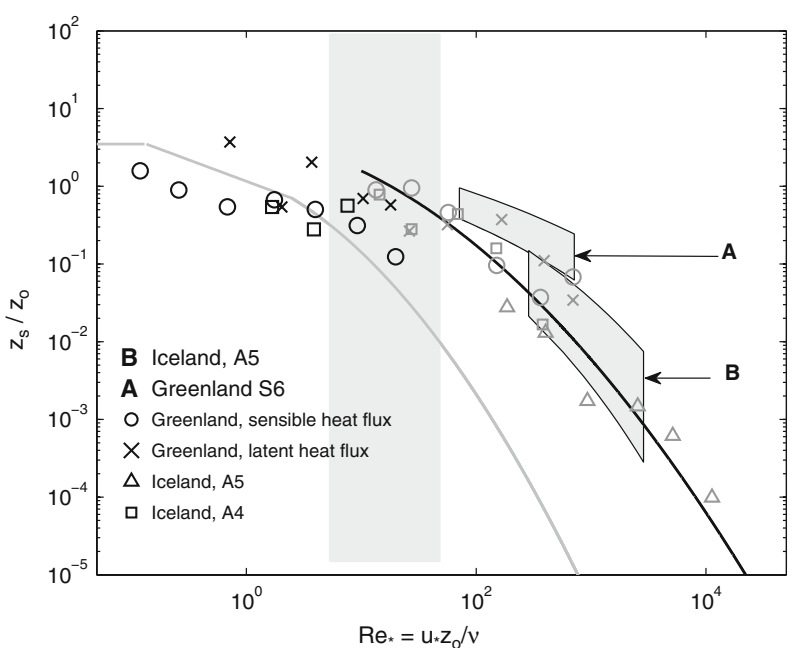

applying $C E=0.75$, the results come close to those from S6 with $C E=0.5$. Furthermore, the size of the ice grains $\left(L_{f}\right)$ is larger at A5 and varying it from 0.005 to $0.03 \mathrm{~m}$ for S6 also shifts the curve downward comparable to the results for varying $C E$.

In summary, the model qualitatively agrees with our hummocky ice results and points out three important parameters for scalar transfer. The extinction parameter $a$ that determines the shape of the exponentially decaying profiles of the various flow parameters in the RSL. It serves as a measure for 'ventilation' efficiency and determines the local velocity scale. Parameter $A$, which estimates the total area of 'active' viscous sublayer per unit volume of air, is difficult to estimate as it involves a whole variety of surface characteristics such as the geometry and size of the hummocks, the ice grain packing density, sheltering efficieny of wakes and the surface area coverage of ice grains with an 'active' viscous sublayer. The size of the ice grains or surface irregularities $\left(L_{f}\right)$ determines the length scale for the bulk transfer coefficient. Furthermore, coefficients $C_{L}, m$ and $n$ are not very well known but, as they scale the efficiency of the bulk transfer description for the surface renewal process, these are also important parameters.

Open Access This article is distributed under the terms of the Creative Commons Attribution Noncommercial License which permits any noncommercial use, distribution, and reproduction in any medium, provided the original author(s) and source are credited.

\section{References}

Abramowitz M, Stegun IAE (1964) Handbook of Mathematical functions. National Bureau of Standard Applied Mathematics Series, 55, Washington, DC

Andreas EL (1987) A theory for the scalar roughness and the scalar coefficients over snow and ice. BoundaryLayer Meteorol 38:159-184

Andreas EL (2002) Parameterizing scalar tranfer over snow and ice: a review. J Hydrol 3:417-432

Andreas EL, Jordan RE, Makshtas AP (2005) Parameterizing turbulent exchange over sea ice: the ice station weddell results. Boundary-Layer Meteorol 114:439-460

Bintanja R, Reijmer CH (2001) A simple parameterization for snowdrift sublimation over antarctic snow surfaces. J Geophys Res 106:31739-31748

Brutsaert W (1975) A theory for local evaporation (or heat transfer) from rough and smooth surfaces at ground level. Water Resour Res 11(4):543-550 
Brutsaert W (1979) Heat and mass transfer to and from surfaces with dense vegetation or similar permeable roughness. Boundary-Layer Meteorol 16:365-388

Brutsaert W (1982) Evaporation into the atmosphere. D. Reidel Publ. Comp., Dordrecht The Netherlands, $299 \mathrm{pp}$

Brutsaert W, Sugita M (1996) Sensible heat transfer parameterization for surfaces with anisothermal vegetation. J Atmos Sci 53(2):209-216

Chamberlain A (1966) Transport of gases to and from grass and grass-like surfaces. Proc R Soc London A 290:236-265

Chamberlain A (1968) Transport of gases to and from surfaces with bluff wave-like roughness. Quart J Roy Meteorol Soc 94:318-332

Danckwerts PV (1970) Gas-liquid reactions. McGraw-Hill, New York, 276 pp

Denby B, Snellen H (2002) A comparison of surface renewal theory with observed roughness length for temperature on a melting glacier surface. Boundary-Layer Meteorol 103:459-468

Fairall CW, Persson POG, Bradley EF, Payne RE, Anderson SP (1998) A new look at calibration and use of eppley precision infrared radiometers. Part I: Theory and application. J Atmos Ocean Technol 15:12291242

Garratt JR (1992) The atmospheric boundary-layer. Cambridge University Press, Cambridge, 316 pp

Grass AJ (1971) Structural features of turbulent flow over smooth and rough boundaries. J Fluid Mech 50:233255

Joffre SM (1982) Momentum and heat transfers in the surface layer over a frozen sea. Boundary-Layer Meteorol 24:211-229

Kanda M, Kanega M, Kawai T, Moriwaki R (2007) Roughness lengths for momentum and heat derived from outdoor urban scale models. J Appl Meteorol Climatol 46:1067-1079

Macdonald RW (2000) Modelling the mean velocity profile in the urban canopy layer. Boundary-Layer Meteorol 97:24-45

Moore CJ (1986) Frequency response corrections for eddy correlation's systems. Boundary-Layer Meteorol 37:17-35

Munro DS (1989) Surface roughness and bulk heat transfer on a glacier: comparison with eddy correlation. J Glaciol 35(121):344-348

Oerlemans J, Björnsson H, Kuhn M, Obleitner F, Palsson F, Smeets CJPP, Vugts HF, de Wolde J (1999) Glacio-meteorological investigations on vatnajökull, iceland, summer 1996: An overview. Boundary-Layer Meteorol 92:3-26

Owen PR (1964) Saltation of uniform grains in air. J Fluid Mech 20(2):225-242

Pandolfo JP (1966) Wind and temperature for constant flux boundary layers in lapse conditions with a variable eddy conductivity to eddy viscosity ratio. J Atmos Sci 23:495-502

Raupach MR, Thom AS, Edwards I (1980) A wind-tunnel study of turbulent flow close to regular arrayed rough surfaces. Boundary-Layer Meteorol 18:373-397

Schotanus P, Nieuwstadt FTM, de Bruin HAR (1983) Temperature measurement with a sonic anemometer and its application to heat and moisture fluxes. Boundary-Layer Meteorol 26:81-93

Shreffler JH (1976) A model for the transfer of gaseous pollutants to a vegetational surface. J Appl Meteorol 15:744-746

Smeets CJPP, Van den Broeke MR (2008) Temporal and spatial variation of momentum roughness length in the ablation zone of the greenland ice sheet. Boundary-Layer Meteorol. doi:10.1007/s10546-008-9291-0

Smeets CJPP, Duynkerke PG, Vugts HF (1998) Turbulence characteristics of the stable boundary layer over a mid-latitude glacier. Part I: A combination of katabatic and large-scale forcing. Boundary-Layer Meteorol 87:117-145

Smeets CJPP, Duynkerke PG, Vugts HF (1999) Observed wind profiles and turbulence fluxes over an ice surface with changing surface roughness. Boundary-Layer Meteorol 92:101-123

Van den Broeke MR, Duynkerke PG, Oerlemans J (1994) The observed katabatic flow at the edge of the greenland ice sheet during GIMEX-91. Glob Planet Chang 9:3-15

Van de Wal RSW, Greuell MR, van den Broeke MR, Reijmer CH, Oerlemans J (2005) Surface mass balance observations and automatic weather station data along a transect near kangerlussuaq, west greenland. Ann Glaciol 42:311-316

Webb EK, Pearman GI, Leuning R (1980) Correction of the flux measurements for density effects due to heat and water vapour transfer. Quart J Roy Meteorol Soc 106:85-100

Wilczak JM, Oncley SP, Stage SA (2001) Sonic anemometer tilt correction algorithms. Boundary-Layer Meteorol 99:127-150

Yang K, Koike T, Ishikawa H, Kim J, Li X, Liu H, Liu S, Ma Y, Wang J (2008) Turbulent flux transfer over bare-soil surfaces: Characterisitcs and parametrisation. J Appl Meteorol Climatol 47:276-290 